\title{
On the Rofe of Refugia in Promoting Prudent Use of Biological Resources
}

\author{
N V Joshi AND MADHAV GADGIL \\ Centerfor Ecological Sciences, Indian Institute of Science, \\ Bangalore 560012 , India
}

Received March 12, 1990

\begin{abstract}
We explore a flnodel of utilization in a premarket economy of a biological resource population by a social group which is the sole owner of the resource. The group is assumed to be motivated to derive as large a harvest as possible while at the same time attempting to keep the risk of extinction of the resource population at a low level It is shown that this can most likely be achieved through total protection of the resource population in parts of its range set aside as refugia Many primitive societies indeed follow this strategy, which deserves to be given more serrous aftention as a tool for the management of renewable resources () 1991 Academic Press, Inc
\end{abstract}

\section{INIRODUCIION}

Pleistocene hunter-gatherers are believed to be responsible for the extinction of a number of larger mammals through overhunting Closet to our times the passenger pigeon was undoubtedly exterminated by overhunting; the same cause has drastically depleted the populations of a wide range of organisms from whales and elephants to dipterocarp trees and orchids (Wilson, 1988; Worster, 1988) At the same time, we know that human societies at all stages of development exhibit practices of restrained resource use that may have the consequence of conservation of resource populations. These range from total protection to certain biological communities as with sacred ponds to closed seasons or mesh size regulations in modern day fisheries In prescientific times such practices were not explicitly stated to pursue conservation objectives, instead they were often cast in the idiom of religious beliefs or social conventions Nevertheless it is plausible that their conservation consequences were not merely incidental (Johannes, 1978; Gadgil and Berkes, 1991). Indeed, in the tribal state of Mizoram in Northeastern India, the indegenous people term the sacred grove from which all harvests are banned as the "safety" forest and the community controlled woodlot from which regulated harvests are made as 
the "supply" forest, suggesting that such prescriptions might have been delibei ately arrived at in the interest $\mathrm{cf}$ resource conservation (Malhotra, 1990)

In this paper we do not enter into the debate as to whether the restraints on resource use by the prescientific societies were in fact functional Instead we take it as a working hypothesis that at least some of the prescientific societies constituted socially homogeneous groups which were both interested in deriving high levels of harvests from a resource population as well as highly averse to the risk of its extermination In other words, we assume that these societies were aiming to arrive and stabilize at a harvesting regime generating maximum sustainable yields (MSY) Such societies of course did not possess the notion of MSY in the modern sense Rather we postulate that they must have been involved in some simple trial and error process that moved toward MSY while keeping the risk of resource population extinction low Such a process can be expected to be a simple feedback process in which the group of harvesters would be using the harvest for acquiring some information toward appropriate adjustment $\mathrm{cf}$ the harvesting effort as well (Mangel, 1985;Walters, 1986) We do not expect it to devote any separate effort toward acquiring such information; and of course such a society would not have at its disposal any elaborate models of dynamics of the resource population Rather it would adjust harvesting effort on the basis of some simple rules of thumb using knowledge cf harvesting effort, harvests realized, and perhaps levels of resource population in their recent past experience We find that strategies of this type, based on assessing the situation over one or a few time steps, fail to converge to that which would give the maximum sustainable yield Convergence is obtained only if any given level of harvesting effort is held steady long enough for the resource population to equilibrate before decisions on adjusting harvesting effort are made Alternatively, if absolute protection is given to the resource in portions of its range, it is often possible to approach the strategy for maximum sustainable yield, We suggest that this is the solution that such human societies are most likely to have hit upon We end by suggesting that refugia or area closures deserve to be considered more carefully as an instrument cf resource management

\section{RESOURCE DYNAMICS}

\section{Biomarr Growth}

We visualize the utilization of a single population of a single resource species by a human group which is the sole owner cf the resource The dynamics of the resource population are specified in terms of a discretetime deterministic model without any age and sex structure Each time step 
consists $\mathbf{r f}$ two phases During the growth phase, the resource biomass $B_{2}$ grows to $B_{t}^{\prime}$ We assume logistic growth, i.e,

$$
B_{t}^{\prime}=r \cdot B_{t}\left(1-B_{i}\right)
$$

Growth is followed by a harvesting phase, where effort $E_{t}$ results in the harvest $H_{i}$ The effort-harvest relationșhip is assumed to fetch diminishing returns and to correspond to the type III or concave resource population profile (Clark, 1985) Thus,

$$
H_{t}=B_{i}^{\prime} \cdot\left[1-\exp \left(-k \quad E_{t}\right)\right] .
$$

Without any loss of generality, we assume $k=1$ throughout After harvest the remaining resource biomass grows in the next time step, Thus,

$$
B_{t+1}=B_{t}^{\prime}-H_{t}=5 ; \exp \left(-E_{t}\right)
$$

and

$$
B_{t+1}^{\prime}=r \quad B_{t+1}\left(1-B_{i+1}\right)
$$

We also explored three alternative forms of population growth, viz ,

Modified logistic: $B_{i}^{\prime}=r B_{i}\left(1-B_{i}\right)^{\gamma}$

Hyperbolic: $B_{t}^{\prime}=r B_{r} /\left(1+(r-1) B_{t}\right)$

Ricker: $B_{i}^{\prime}=B, \exp \left[r\left(1-B_{i}\right)\right]$

and an alternative form of effort-harvest relationship

$$
H_{t}=\frac{B_{t}^{\prime} E_{t}}{1+E_{t}}
$$

However, we found the results of all these models to be very similar We therefore describe only the results of the models. described by Eqs (1) and (2)

\section{Constant Harvesting Effort}

Let us begin by assuming that the harvesting effort $E$ is held constant Then it is obvious that the resource population must reach an equilibrium value given by

$$
1-\frac{1}{r \exp (-E)}
$$

The resource persists as long as the harvesting effort is within the limit,

$$
E<E_{\max }=\ln r
$$


The equilibrium value of the resource "population is a monotonically decreasing function of $E$, while the harvest vanishes at points $E=0$ and $£=E_{\max }$ In the case of our model, the harvest attains a maximum value of

$$
\hat{H}=\frac{(r-1)^{2}}{4 i}
$$

when

$$
\hat{E}=\ln \frac{(1+r)}{2}
$$

\section{(see Fig 1)}

'Thequestion which we now take up is: What sort of harvesting strategy would allow a community to "home in" on $E$ ?

\section{Variable Harvesting Strategies}

We assume that the human group which is the sole owner of the resource is a co-operative group which makes decisions in group interest as perceived at any given time (Berkes, 1989) We do not postulate any saturation of the demand as harvests increase, and the group is assumed to attempt as large a harvest as possible 'Tobegin, we ignore all costs such as search, harvesting, and processing costs The owner group is assumed to be in possession of information on harvesting effort put in and harvests realized at all points of time Given this, we expect it to keep adjusting the harvesting effort until harvests as high as possible are obtained

The basic decision that a harvester would lace is whether to decrease, maintain at the same level, or enhance the harvesting effort from one time

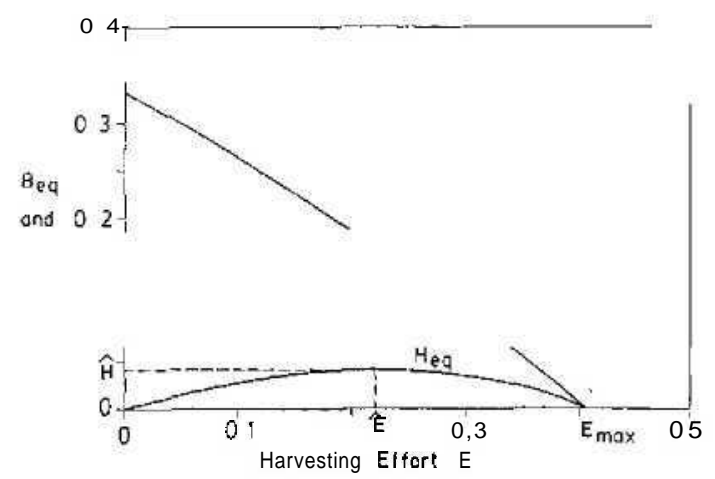

FIG 1 The equilibrial values cf resource population and harvest as a function of harvesting effort that is being held constant when there is no Iefugium $(r=15)$ 
interval to the next, The simplest decision-making procedure for a harvester group following such a feedback strategy may then be specified as:

1 If an increase in effort has led to an increase in harvest. then increase the effort further,

2 ff an increase in effort has' led to a decrease in harvest, then decrease the effort

3 If a decrease in effort has led to an increase in harvest, then decrease the effort further

4 ff a decrease in effort has led to a decrease in harvest, then increase the effort

In a discrete time model, $\mathrm{F} D(t)$ is the change in the effort so that $E(t+1)-E(t)+D(t)$, then, one expression of the above prescription would be

$$
D(t)=x D(t-1) \quad \text { if } \quad H(t)>H(t-1)
$$

and

$$
D(t)=-x \quad D(t-1) \quad \text { if } \quad H(t)<H(t-1),
$$

where $x$ is some positive scale factor $x$ could be constant or variable (say, proportional to the incremental increase in the harvest relative to the increase in the effort)

$$
\frac{H(t)-H(t-1)}{E(t)-E(t-1)}
$$

\section{IMPLICATIONS}

\section{Run-Away Increases}

On account cf the decision rule (4), there is a clear tendency for the harvesting effort to increase till the resource population is eventually wiped out, This is shown in Fig $2 \mathrm{a}$ when the increment or decrement in $£$ is fixed, and in Fig 2b, when the increment or decrement is variable,

This unchecked increase in effort is a consequence of the following fact When harvesting effott can change at each step, the harvest obtained by enhancing the effort is always higher than that which can be sustained at the previous level of effort This is because the resource capital starts getting used up. Furthermore, even when the harvesting effort has reached levels above $\hat{E}$, that constant effort at which sustained harvest is maximized, a 

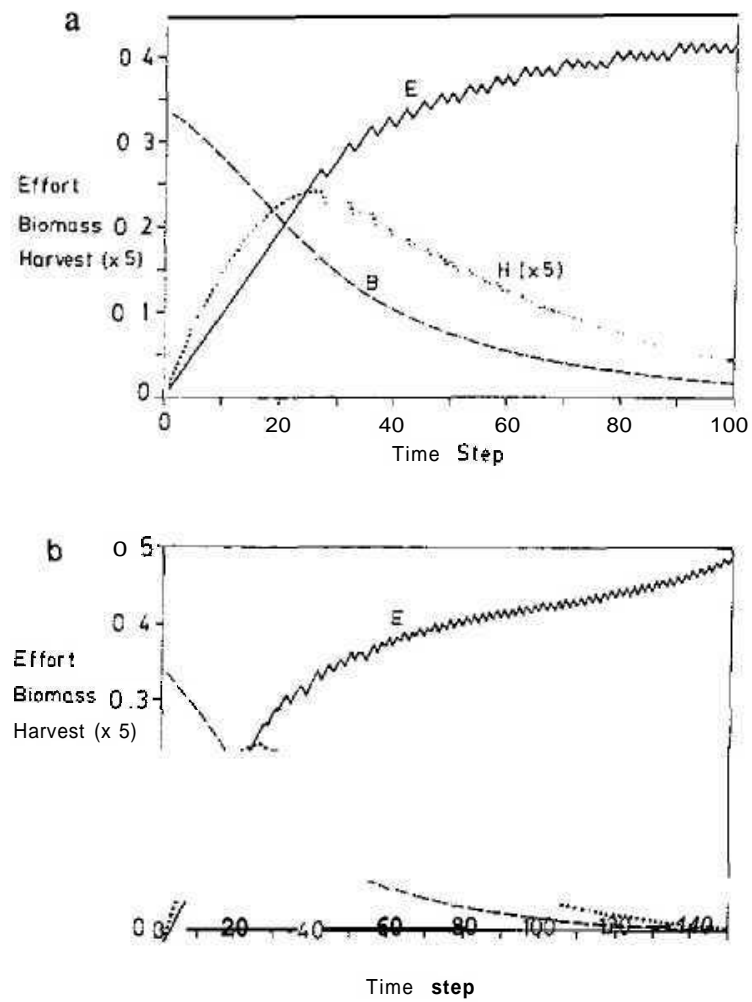

FIG 2 Variation of harvesting effort (-) biomass level $(-)$ and the harvest obtained ( ) as a function of time when harvesting effort is adjusted at every time step $(r=15)$ (a) When the change in effort consists of a fixed increment or decrement $=001$ (b) When the change in effort is variable, being proportional to the ratio of change in harvest obtained relative to the previous change in the effort (constant of proportionality $=1$, maximum permitted change in the effort $=0.01$ )

decrease in harvesting effort does not immediately result in an enhancement in the harvest (Fig 3) Hence the effort continues to drift to higher and higher values till such times as the resource capital is exhausted

\section{Other Outcomes}

There are three modifications of the model which can prevent such run-away increases in harvesting effort

(1) No change in harvesting effort: We assume no change in harvesting effort if the change in harvest is below a prespeciffed threshold Then, depending on the initial conditions, the harvesting effort stabilizes at 


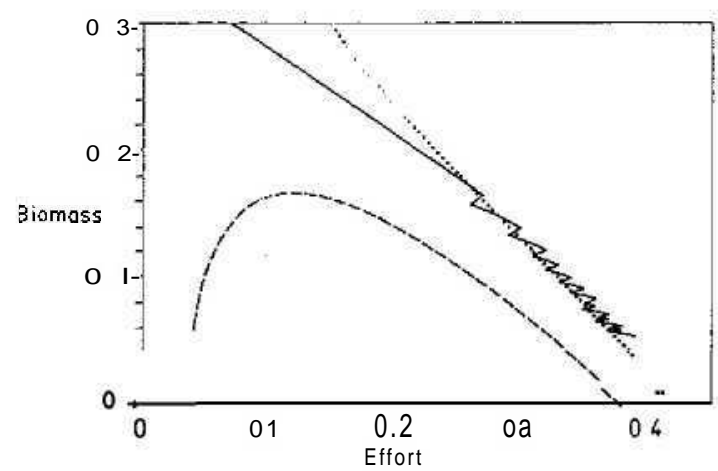

FIG 3 Regions in the space of biomass level and harvesting effort where increasing effort leads to decreasing harvests (above the dotted line) and where decreasing effort leads to increased harvest (below the dashed line) for the strategy where effort is adjusted in every time step by a fixed amount The trajectory (solid line) indicates how the effort gradually increases, eventually resulting in decimation of the resource $(r=15)$;

different values. However, most of these final states are far from $\hat{E}$, the constant effort level corresponding to maximal sustainable yield

(2) Biomass dependent harvests: We have also explored strategies wherein the decision to increase or decrease the effort was based on both the harvest and the standing stock of resource biomass All these strategies lead either to run-away harvesting, or to the effort continuing to decrease to very low levels of harvest Again there is no approach of harvesting effort to $\hat{E}$

(3) Harvests imply costs: We have also investigated the consequences of considering costs associated with harvesting effort A linear cost function was used, with cost per unit effort being denoted by $c$, so that the harvest obtained is given by

$$
H_{t}=\mathrm{fi} ;\left[1-\exp \left(-E_{t}\right)\right]-c E_{t}
$$

Any nonzero value of $c$ automatically puts an upper limit on $E$, smaller than $E_{\max }$. High values of $c$ imply that the resource would always be consumed at very low levels and the resource population is in no danger of decimation We can also define for this case an "optimal" harvesting effort such that sustained benefit minus cost is maximized when that effort is maintained constant. Application of decision rule (4) in this context does not lead to the effort approaching this optimat level For low values of $c$ the model exhibits irregular cycles, with the biomass maintained at low levels for long stretches of time These are separated by short periods of decrease in the effort and recovery of the resource The mean value of the 
harvest, however, is always smaller than that corresponding to the maximum sustainable yield at constant effort

\section{Assessing Sustainable Harvests}

Thus, a procedure dependent on information on the relationship between harvesting effort and harvests when the latter have not equilibrated with respect to the former does not permit the harvester to stabilize harvesting effort at that level which yields maximum sustainable yields MSY can be reached if the harvester has access to information regarding the relationship between harvesting effort and harvests al equilibrium, which in turn can only be obtained by holding the harvesting effort constant at a
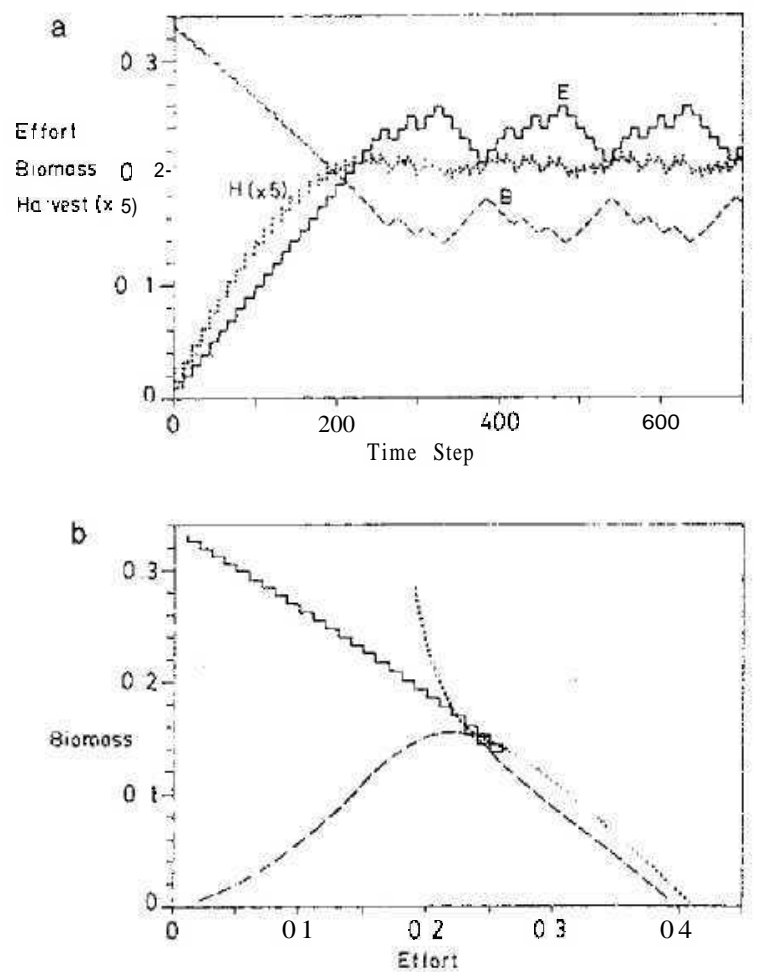

FIO 4 (a) Variation of harvesting effort $(-)$, biomass level $(-)$ and the harvest obtained ( ) as a function of time when harvesting effort is adjusted at every 10thtime step; with fired increment or decrement $(l=15, D=001)$ (b) Regions in space of biomass level and harvesting effort such that increased effort leads to decreased harvests (above the dotted line) and where decreased effort leads to increased harvest (below the dashed line) for the above strategy Due to the closeness of the two boundaries, the trajectory (solid line) does not meander to increasingly high efforts but oscillates near regions corresponding to maximum sustained yield 
series of different levels. Therefore, the decision rule employed by the harvester must not allow the harvesting effort to be continually changed from one time interval to the next; the effort must be maintained at a fixed level even if the harvest is declining, Such a decline occurs because less and less resource capital above the equilibrium level will be available for consumption while the effort remains steady, For the effort and harvest to equilibrate and remain at that level in a constant environment will take some time, This process will of course be more complicated in a fluctuating environment: there the harvest-effort relationship will bnly gradually settle down to the one around the mean equilibrial relationship

Is there an algorithm that can guarantee that the harvesting effort stabilizes at levels close to the optimal through a simple feedback process such as envisaged here? The answer is yes, if the harvester holds the effort steady for several time intervals and then compares the harvests and efforts at the end of such a period with harvests and efforts at the ends of one or more previous periods of steady effort, Applying an analogue of decision rule (4) to such a "multiple-step-wait, last-step-compare" strategy can in fact lead the harvester to the optimal effort (see Fig. 4a and b), It should be noted that when the harvesting effort is held constant, using the information from only the last step in a multiple step-wait procedure is more effective than averaging the information from all time steps, This is because the earlier time steps include transients further from the sustained harvest relationships and merely distort the estimate

In real-lie situations harvesters must frequently operate on the basis $\mathbf{r f}$ partial information about the relationship of harvest to effort (Clark, 1985; 1990; May, 1984; Walters, 1986), With fir less known of the dynamics of biological populations than is the case today, harvesters would have had to employ a feedback process analogous to the one sketched above, Under these circumstances very long waits holding the effort constant, and then careful adjusting of the effort based on some much earlier information would be difficult to implement. By and large then the societies would have to follow a decision rule such as (4) considered above, As we have seen, the result would be a harvesting effort drifting to higher and higher levels or fluctuating wildly with eventual extermination of the resource population becoming a highly probable event Alternatively the effort could stabilize at some arbitrary level of harvesting effort not necessarily close to $\hat{E}$ Clearly, it would be worth exploring a qualitatively different approach,

\section{REFUGIA}

There is considerable empirical evidence that while some prescientific societies decimated their resource base, others did manage to exist in 
equilibrium with their environment This was particularly true of sedentary, territorial groups in stable productive environments such as tropical humid forests or coral reef islands Occasionally such an equilibrium may have been reached because the demands of these human groups on the resource populations were too low to seriously affect them However, there is evidence that human groups responded to depletion of resource populations by setting up restrictions on resource use One such significant measure involves conferring total protection to part of the resource population in refugiasuch as sacred groves, sacred ponds, or sacred areas on the sea coast (McNeely and Pift, 1985; Ruddle and Johannes, 1985), Thus, a fraction of the resource population would be maintained totally free from harvesting There were invariably a multiplicity of these refugia of small and moderate sizes so that the harvested and protected populations would be fairly well mixed

Our basic model can be extended to include a refugium without changing the dynamics of growth Eq (1) We now assume that regardless of the total biomass a fraction $a$ is always immune from harvesting. Only the remaining fraction of population, $(1-\underline{x})$, lying outside the refugium patches is exploited Then, with the notation as before the harvest $\boldsymbol{H}$, is given by

$$
H_{t}=(1-\underline{\alpha}) B_{\prime}^{\prime}\left\{1-\exp \left(-E_{t}\right)\right\}
$$

and the biomass remaining after exploitation equals

$$
(\mathrm{I}-\alpha \jmath) B_{\imath}^{\prime} \exp \left(-E_{t}\right)+\underline{\alpha} B_{\imath}^{\prime}
$$

We assume complete mixing between the nonharvested population in the refugium and the harvested population outside during every growth phase The resource biomass at the beginning of the next time step is thus given by

$$
\begin{aligned}
B_{t+}, & =\underline{\alpha} \quad I \quad B_{t}\left(1-B_{t}\right)+(1-\underline{\alpha}) r B_{t}\left(1-B_{t}\right)\left\{\exp \left(-E_{t}\right)\right\} \\
& \approx r \cdot B_{t}\left(1-B_{t}\right)\left\{\underline{\alpha}+(1-\underline{\alpha}) \exp \left(-E_{t}\right)\right\}
\end{aligned}
$$

When the harvesting effort is held constant in time, the resource population must reach an equilibrium value given by

$$
B_{\text {eq }}=1-\frac{1}{r\{\underline{\alpha}+(1-\underline{\alpha}) \exp (-E)\}}
$$


Hence a nonzero level of the resource population will be maintained $\mathrm{F}$

$$
r\{\underline{\alpha}+(1-\underline{\alpha}) \exp (-E)\}>1
$$

Thus, when the refugium is sufficiently large, i e, a $>1 / r$, the resource population will persist regardless of the level of harvesting effort Figure 5 shows the equilibria] resource population levels and harvests for different values of refugium size when the harvesting effort is infinitely high For this case

$$
B_{\mathrm{eq}}=1-1 /(r \underline{\alpha})
$$

and

$$
H_{\text {eq }}=\frac{(1-\underline{\alpha})(r \underline{\alpha}-1)}{r \underline{\alpha}^{2}}
$$

The $\hat{\alpha}$ that maximizes this Hequals $\left\{2 /\left(1 \dot{\boldsymbol{T}}_{\mathbf{r}}\right)\right\}$ andatthis level $\boldsymbol{H}$ equals $(\mathrm{r}-1)^{2} / 4 \mathrm{r}$ Note that this maximum is the same as that obtained in the absence of a refugium This conclusion is of course dependent on the assumption of complete mixing In the absence cf such mixing the maximum sustainable yield obtained in the presence cf a refugium would be lower It is notable that the refugia in the form cf sacred groves or ponds are not a system cf just a few large ones but rather of numerous, highly dispersed small elements (Gadgil and Vartak, 1976) Such a spatial

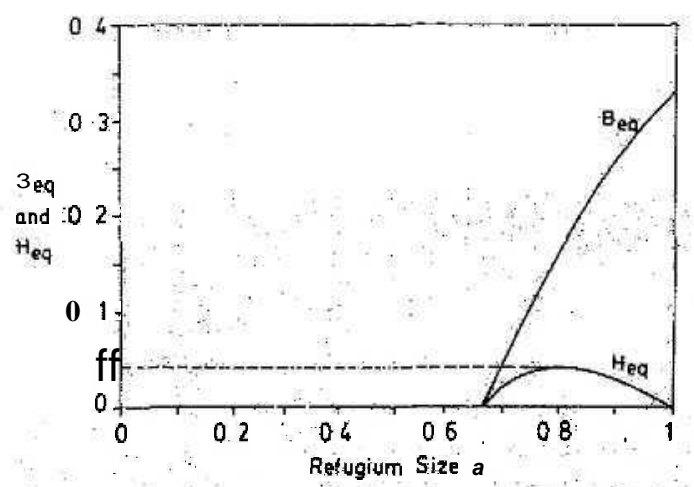

FIG. 5 Ihe equilibrial values of resource population and harvest as a function of refugium size when the harvesting effort is infinitely large $(r=15)$ 
distribution of refugia wculd tend to ensure, rather complete mixing of populations inside and outside the refugia

If the harvesting effort does not grow infinitely large, protecting a fraction smaller than a would be sufficient to guarantee resouice security Furthermore, given complete mixing of harvested and protected resource populations, the sustainable larvest obtained at many combinations of harvesting effort and refugium size can be as large as the maximum sustainable harvest obtained i 1 the absence of a refugium (Fig 6) In other words there is a whole serie; of combinations of a and $E a$ which the sustained harvest reaches the maximal sustainable level of $(r-1)^{2} / 4 r$ These combinations satisfy the relation

$$
\underline{\alpha}=\frac{\frac{2}{r+1}-\exp (-E)}{1-\exp (-E)}
$$

In conclusion, the maximum sustained harvest is not diminished by giving total immunity to a part of the resource population from pressures of harvesting provided that the refugia are adequately dispersed in space It is obvious that a firm prohibition against harvesting from a set of localities is easier to implement than a quantitative restriction on harvesting effort. The provision of refugia can therefore be a highly effective means rf ensuring sustained harvests from a biological resource

\section{Adjusting Refugium Size}

As before, we must enquire into the kind of decision rules that may be employed to adjust simultaneously refugium size and harvesting effort Since the feedback strategy aims to reduce the risk of resource extermination, the procedure would involve an increase in the area under a refugium or a set of refugia whenever the harvesting effort is stepped up The deci-

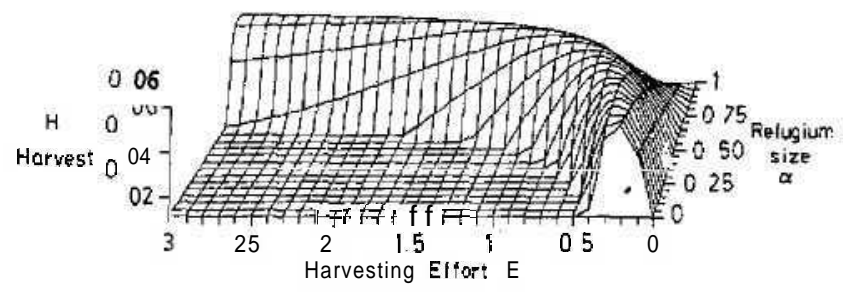

FIG 6 Ihe relationship between equilibrial values of harvest, harvesting effort, and refugium size $(r=15)$ 
sion rule (4) may then be amplified by adding to a change in harvesting effort a change in refugium size as

$$
\alpha=z \quad D,
$$

where 2 is a positive constant Then, if the initial harvesting effort and refugium size are $E_{0}$ and $\underline{\alpha}_{0}$, they would at any time be related by

$$
\underline{\alpha}_{t}=\underline{\alpha}_{0}+\mathrm{Z} \quad\left(E_{i}-E_{0}\right)
$$

subject to the constraint $0 \leqslant \mathrm{a} \leqslant 1$ Correspondingly the effort would be constrained to lie in some range $E_{\min }$ and $E_{\max }$ If the effort is held constant at some $E, E_{\min }<E<E_{\max }$, then the equilibrium value of the resource population $B_{\mathrm{eq}}$ is given by

$$
B_{e s}=1-\frac{1}{r\left\{\underline{\alpha}_{0}+z\left(E-E_{0}\right)+\left(1-\underline{\alpha}_{0}-z\left(E-E_{0}\right) e^{E}\right\}\right.}
$$

The significant questions then are: Does the amplified decision rule (4) with (5), (a)reduce the risk of resource decimation, and (b) render it more likely that the harvest stabilizes close to the maximal sustainable harvest ${ }^{\text {") }}$ It is evident that this new procedure, which does not invoke long waits with $E$ and a held constant, would still not provide the harvester with an accurate assessment of the relation between effort, refugium size, and harvest In the absence of such information. there is no guarantee of the harvester reaching the maximal sustainable harvest, However, this algorithm does modify the previously expected outcome in another important way In the absence of a refugium the cause of continuing drift of harvesting effort toward higher values was that any increase in harvesting effort was rewarded by nonsustainably higher harvests, This is no longer the case here because every increment in the harvesting effort is accompanied by an increase in the refugium size, thus providing an additional protection to the resource capital If the constant $z$ in decision rule (5) is not too small, i e, a refugium or a set of refugia of a reasonable size is obtained, an increased harvesting effort is prevented from eating excessively into the resource capital 'The tendency of the harvesting effort to continually drift to higher and higher values is therefore checked and as Fig $7 \mathrm{a}$ and $7 \mathrm{~b}$ show, $E$ and a do tend to stabilize at moderate levels

The refugium system therefore definitely reduces the risk of decimation of the resource In addition, it also facilitates the harvester reaching a harvest not far from the optimal under a wide range of parameters When faced with inadequate information, then, a decision rule analogous to (5) is an effective way of managing a biological resource population 

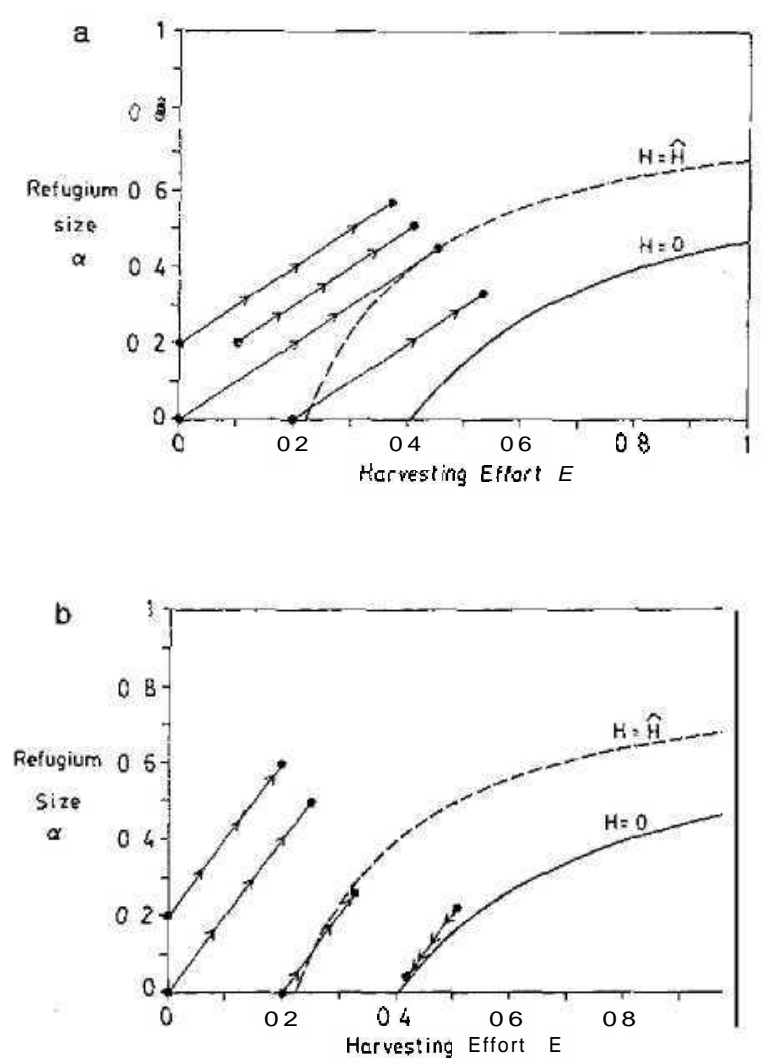

FIG. 1 Irajectory corresponding to the simultaneous changes in the harvesting effort $£$ and the refugium size $a$ The initial and final (equilibrium) point of each trajectory is designated by filled circles Some trajectories end close to the combination of $(\underline{\alpha}, E)$ corresponding to maximum sustained harvest, designated by a dashed line (a) $r=15, Z=I$ (b) $\mathbf{r}=15, Z=2$

\section{Temporal Refugia}

A refugium in space prevents the continual drift of harvesting effort to higher and higher levels by denying access to a laxger fraction of the resource capital whenevex the haxvesting effort is stepped up. Conversely, it also opens up access to a larger fxaction of the resource capital whenever the haxvesting effort is stepped down This counteracts the tendency of evex higher harvesting effoxts being encouxaged because they eat, albeit nonsustainably, into the resource capital A refugium in time, i e., a period over which all haxvest is halted, could act in a similar fashion if the length of 
such a period relative to the length of period over which harvest is permitted was increased every time the harvesting effort is stepped up, and vice versa

Figure 8 depicts sustained harvest as a function of harvesting effort and the ratio of length cf periods of rest $(V)$ andharvest $(U)$ when these are held constant, As in the case of spatial refugium, there are several combinations of harvesting effort and the remporal refugium for which harvests equal or close to maximal sustainable harvest of $(r-1)^{2} / 4 r$ can be realized However, unlike in the case of spatial refugium, there can be no value cf $(V / U)$ which can guarantee against resource extermination as the harvesting effort approaches infinity In that sense a temporal refugi.m is not as effective as a spatial refugium in guarding against resource extermination,

\section{Optimizing the Temporal Refugium}

We have investigated the possibility of arriving at a decision rule which would lead to stabilization of the harvesting effort and periods of re it and harvest at values which generate harvests equivalent to or approaching the maximal sustainable harvests This is difficult, at least in the case of the discrete time model being explored here, As before, let $U$ be the length of period $\mathbf{r f}$ harvest, and $V$ the length of period of no harvests Let $r$ be the current cycle of harvest and rest and $(\mathbf{7 - 1})$ the previous cycle. $E(T)$ is the harvesting effort held constant over the period $U$ of the cycle $T, \boldsymbol{H}$ the total harvest obtained over the period of harvest $U$, and $H^{\prime \prime}=H^{\prime} /(U+V)$ the average harvest over a cycle of harvest and rest Then the decision rule becomes

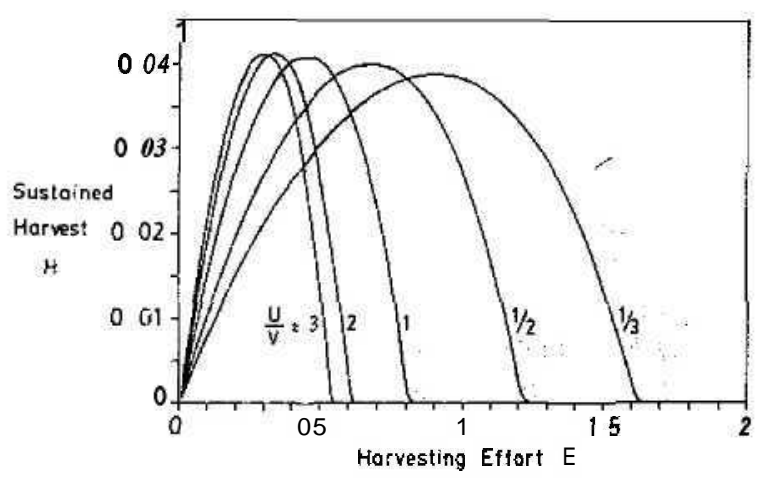

FIG 8 Sustained harvests as a function of harvesting effort (E) for differentcombinations of the lengths of periods of rest $(V)$ and harvest $(U]$ when all the three are maintained constant $(r=15)$ 


$$
\begin{gathered}
D(T)=x D(T-1) \quad \text { if } \quad H^{\prime \prime}(T)>H^{\prime \prime}(T-1) \\
D(T)=-x \cdot D(T-1) \quad \text { if } H^{\prime \prime}(T)<H^{\prime \prime}(T-1) \\
\left.\begin{array}{c}
E(T+1)=E(T)+D(T) \\
V(T+1)=V(T)+Z^{\prime} \mathrm{D}(7) \\
U(T+1)=U(T)-Z^{\prime \prime} \quad D(T)
\end{array}\right\}
\end{gathered}
$$

The decision rule (6) must operate under the constraint that $U$ and $V$ can only take integral values We have considered three possible ways in which the ratio $V / U$ can be adjusted: by changing only $U$, only $V$, or both simultaneously, In the first case, with length of rest interval held constant, the length of harvest interval continues to increase indefinitely with resource extermination the final consequence In the second and the third cases, where the length of rest interval can change, it keeps oscillating between 0 and 1 and the average harvest continues to fluctuate with no tendency to approach maximal sustainable levels In general the temporal refugium solution appears less likely as a guard against resource extermination. Neither is it likely to permit harvests close to maximal sustainable levels In terms of implementation as well, it is more difficult, since it means that resources would be totally unavailable for some extended period: a regulation less likely to be acceptable than protecting a fraction cf the resource population $\mathrm{Cf}$ course, refugia in time (such as closed seasons) and combinations cf spatial and temporal refugia (such as rotational grazing practices) have been elements of resource conservation practices However, experience of modern fishery management suggests that closed seasons typically lead to situations cf overcapacity, very brief fishing seasons, and in some cases (eg, Peruvian anchoveta) ultimately to the collapse of the fishery (Clark, personal communication),

\section{DISCUSSION}

Many prescientific societies exhibit a wide range $\mathrm{cf}$ practices $\mathrm{cf}$ restrained resource use Gadgil and Berkes (1991) examine the nature of these ptactices in some depth and conclude that it is likely that a significant proportion of such practices must have evolved through a process of trial and error to sustain resource use in spite of high levels of demand Systems of many, small and dispersed, or plentifully patchy (Quitin and Hastings, 1987) spatial refugia such as sacred groves, sacred ponds, or sacred stretches cf sea coast may have been one such a resource use system with a positive consequence These systems combined the function of conservation and sustainable use, and as argued above may have evolved through a simple trial and error process Remnants of such systems still persist in many parts of old world tropics and on Pacific islands and continue to play 
a useful role, Indeed the only surviving population of a turtle, 7rionyx nigricans persists in a sacred pond in Bangladesh (Reza Khan, 1980)

Europe too once harbored many such refugia (Frazer, 1922) These were among the Pagan practices first rejected by Christianity and later by the scientific world view, The patterns of biological resource use that followed proceeded, in words of Walters (1986), more like mining operations, with a boom followed by stock depletion and collapse; either no thought was given to long term, ot the resources were considered inexhaustible Over this period of last few centuries of European domination, little attention was paid to conservation of biodiversity either, except through hunting preserves of nobles, and other forms of game parks (Gadgil, 1987), Within the last century however there has been a dramatic shift in viewpoint with emergence of theories about the limits cf sustainable harvests and beginning of public regulation of harvesting activity At the same time has emerged a movement to conserve wilderness and biodiversity through systems of large scale spatial refugia such as National parks There has thus developed a dichotomy between systems of conservation and sustainable resource use The experience of these systems bas brought to light several limitations (Clark, 1985; 1990; Soule, 1986; Walters, 1986) These include a great paucity of information on the behavior of target populations, interactions among many species, the changing behavior of human populations acting either as harvesters or as protectors, and the many uncertainties in the real world, Many new approaches are coming up in response to these problems These include the development of an adaptive management process similar to the trial and error process sketched above, although the models being investigated are of course far more sophisticated (Holling, 1978; Walters, 1986) These also include the emergence $\mathrm{cf}$ a discipline of conservation biology (Soule, 1986) Among the liveliest debates of conservation biology is that on the merits and demerits of different patterns of spatial dispersion of refugia; few large, many small, or plentifully patchy (Quinn and Kastings, 1987; Soule and Simberloff, 1986),

We believe that traditional systems of plentifully patchy orefugia carry useful lessons as well as have a practical role in the present day context They are, first, relevant in suggesting that such systems, implicit in fishery zones or more explicitly considered as area closures could play a more positive role in management of renewable resources and deserve to be considered futher in some depth (Clark, 1985; Walters, 1986) Second, they suggest that plentifully patchy nature reserves, an option that brings the experience of wilderness with its transformative value (Norton, 1987) to everyone's doorsteps, should be more seriously explored by those committed to conservation of biodiversity, Third, and perhaps most important, they suggest that it is possible to combine the two objectives of conservation and sustainable use in a meaningful fashion (Reid and Miller, 1989) 
TABLE I

Symbols Used in the Text

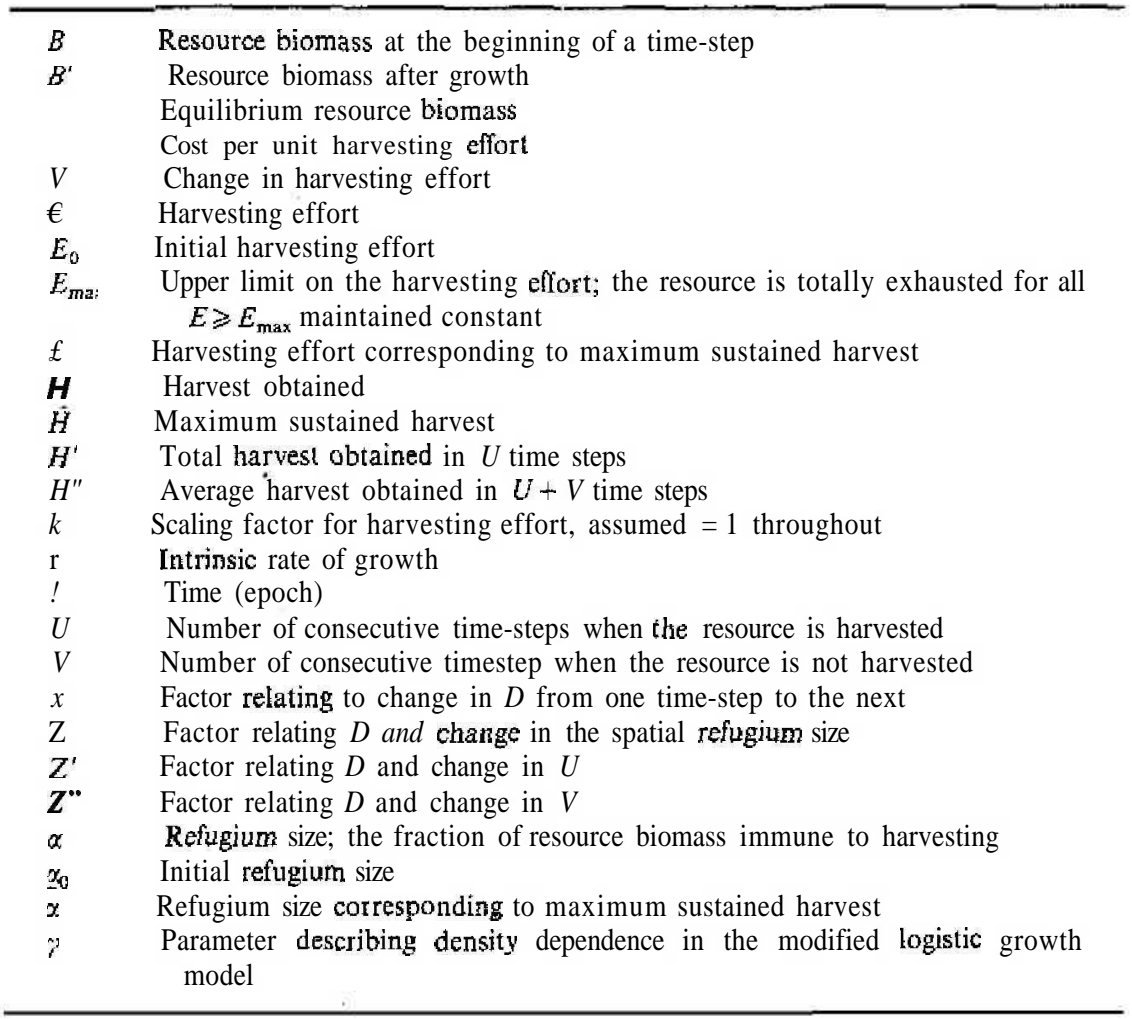

\section{ACKNOWLEDGMENTS}

We thank Marcus Feldman, Sulochana Gadgil, Anil Gore, Sharad Lele, Vidyanand Nanjundiah, and S D Tuljapurkar for long and lively discussions and many useful ideas and Colin W Clark for very helpful comments This work was supported by grants from the Ministry of Science and Technology, and Ministry of Environment and Forests, Government of India

\section{REFERENCES}

Berkes, F , Ed 1989 "Common Property Resources: Ecology and Community Based Sustainable Development," Belhaven Press, London

CLARK, C W. 1985. "Bioeconomic Modelling and Fisheries Management," Wiley, New York CLARK, C W. 1990 Mathematical file-economics: The Optimal Management of Renewable Resources," 2nd ed. Wiley, New York

CLARK, C W, Personal Communication 
FRAzER, J G 1922 “The Golden Bough: A Study in Magic and Religion," Macmillan, London.

GADGIL, M 1987 Diversity: Cultural and biological, Trends Ecol Evol 2(12), 369-373,

GADGIL, M , AND BERKEs, F (1991). Traditional resource management systems, Resource Management and Optimization 18, 127-141

GaDGIL, M., AND VARTAK, V D 1976, Sacred groves ofWestern Ghats cf India Econ Bot $30,152-160$.

Holling, C S., Ed 1978 Adaptive environmental assessment and management, in "Wiley International Series on Applied Systems Analysis," Val 3, Wiley, Chichester,

JOHANNES, R E 1978. Traditional marine conservation methods in Oceania and their demise, Ann Rev System Ecol 9,349-364,

MaLhotRA, K C 1990 Village supply and safety forest in Mizoram: A traditional practice of protecting ecosystem, in "Abstracts of V International Congress of Ecology," p 439

MANGel, M 1985 "Decision and Control in Uncertain Resource Systems," Academic Press, London.

MAY, R , Ed 1984 "Exploitation of Marine Communities," Springer-Verlag, Berlin

McNeElY, J A., AND PIFT, D., Eds 1985 "Culture and Conservation: 'The Human Dimension in Environmental Planning," Croom Helm, Dublin

NORTON, B. G 1987. "Why Preserve Natural Variety?" Princeton Univ. Press, Princeton, NJ

Quin, J F , AND Hastings, A 1987 Extinction in subdivided habitats, Conserv Biol 1, 198-209.

REID, W C V , AND MILLER, K R 1989 "Keeping Options Alive: 'The Scientific Basis for the Conservation of Biodiversity," World Resources Institute, Washington, DC

REZA KHAN, M A 1980 'Theholy turtle of Bangladesh Hormbill 4,7-11

RUDDLE, K , AND JoHANNes, R E ,Ed. 1985 "The Traditional Knowledge and Management cf Coastal Systems in Asia and the Pacific," UNESCO, Indonesia.

Soul., M E , Ed 1986, "Conservation Biology: The Science of Scarcity and Diversity," Sinauer Associates, Inc, Sunderland, MA.

SOULE, M E , AND SIMBERLOFF, D. 1986 What do genetics and ecology tell us about the design cf nature reserves? Biol Conserv 35, 19-40

Wariers, C 1986, "Adaptive Management cf Renewable Resources," Macmillan, New York

WILSON, E O , Ed 1988 "Biodiversity," National Academic Press, Washington, DC

Worster, D., Ed 1988 "The Ends of the Earth: Perspectives an Modern Environmental History," Cambridge Univ Press, Cambridge, 